\title{
Theoretical and technical advancements of near infrared spectroscopy and its operational impact in industry
}

\author{
C.W. Huck \\ Institute of Analytical Chemistry and Radiochemistry, CCB - Center for Chemistry and Biomedicine, Innrain 80/82, 6020 Innsbruck, Austria. \\ E-mail: christian.w.huck@uibk.ac.at
}

In the industrial environment, near infrared spectroscopy (NIRS) on one hand enjoys increasing popularity due to its defined advantages over classical analytical techniques (e.g. chromatography) including fast, non-invasive and simultaneous determination of several physical and chemical parameters. On the other hand, hardly any other analytical discipline is making such fast and fundamental technical and theoretical advancements. Miniaturization of spectrometers down to light weight portable devices is achieved via micro-electro mechanical systems (MEMS) or linear variable filter (LVF) instruments, operating with restricted performance in a defined wavenumber range. Fast NIR imaging devices are becoming popular checking conformity and spatial distribution of ingredients. NIRS is also benefiting from theoretical advances including design of experiment (DOE) for systematic optimization purposes, special algorithms for calibration transfer between different types of spectrometers, and last but not least, quantum chemical approaches are becoming essential for distinct band assignments especially in case of complicated combination and overtone vibrations. Care must be taken about how these advancements can be successfully applied in the industrial environment.

\section{Introduction}

The number of publications dealing with "infrared spectroscopy" has been linearly increasing during the last decade. Performing a SciFinder database search of the American Chemical Society (ACS) shows that between 2006 and 2016 the total number of published manuscripts increased from 1000 to 2500. One reason for this increase is the fact that the several advantages of NIRS get more and more appreciated also by the industry: fast analysis, high-sample throughput, simultaneous determination of chemical/physical parameters, ease of application, possibility to use portable instruments, cost reduction etc. Another reason is the two main food scandals, the milk and horse meat scandals in 2008 and 2013, respectively, which pointed out clearly the necessity to develop a fast and powerful analytical technology for quality control. During the milk scandal China reported an estimated 300,000 victims in total. Six infants died from kidney stones and other kidney damage with an estimated 54,000 babies being hospitalized. ${ }^{1,2}$ During the horse meat scandal, of 27 beef burger products tested, 37\% were positive for horse DNA, and $85 \%$ were positive for pig DNA. ${ }^{3}$ Food fraud in general has been shown to be a serious problem urgently demanding efficient quality control tools. ${ }^{2-4}$ From these dramatic circumstances it becomes clear that there is a permanent demand for innovations in analytical chemistry in order to increase efficiency, in terms of speed of analysis enabling high sample throughput, sensitivity to improve the detection limit and selectivity to find the needle in the haystack (Figure 1). One attempt to improve efficiency is to link NIR spectroscopic data with highly potent other analytical (reference) methods: Solid-phase extraction (SPE), miniaturized liquid and electrophoretic separation techniques $(\mu \mathrm{LC}, \mathrm{CE})^{6,7}$ and mass spectrometric $(\mathrm{MS})^{8,9}$ methods allow to achieve some of these aims. From the technical point of view, there are currently two mainstream trends in analytical

\section{Correspondence}

C.W. Huck (christian.w.huck@uibk.ac.at)

doi: $10.1255 /$ nir2017.057

Citation: C.W. Huck, "Theoretical and technical advancements of near infrared spectroscopy and its operational impact in industry", in Proc. $18^{\text {th }}$ Int. Conf. Near Infrared Spectrosc., Ed by S.B. Engelsen, K.M. Sørensen and F. van den Berg. IM Publications Open, Chichester, pp. 57-64 (2019). https://doi. org/10.1255/nir2017.057

\section{(c) 2019 The Author}

This licence permits you to use, share, copy and redistribute the paper in any medium or any format provided that a full citation to the original paper is given, the use is not for commercial purposes and the paper is not changed in any way.

ISBN: 978-1-906715-27-4 


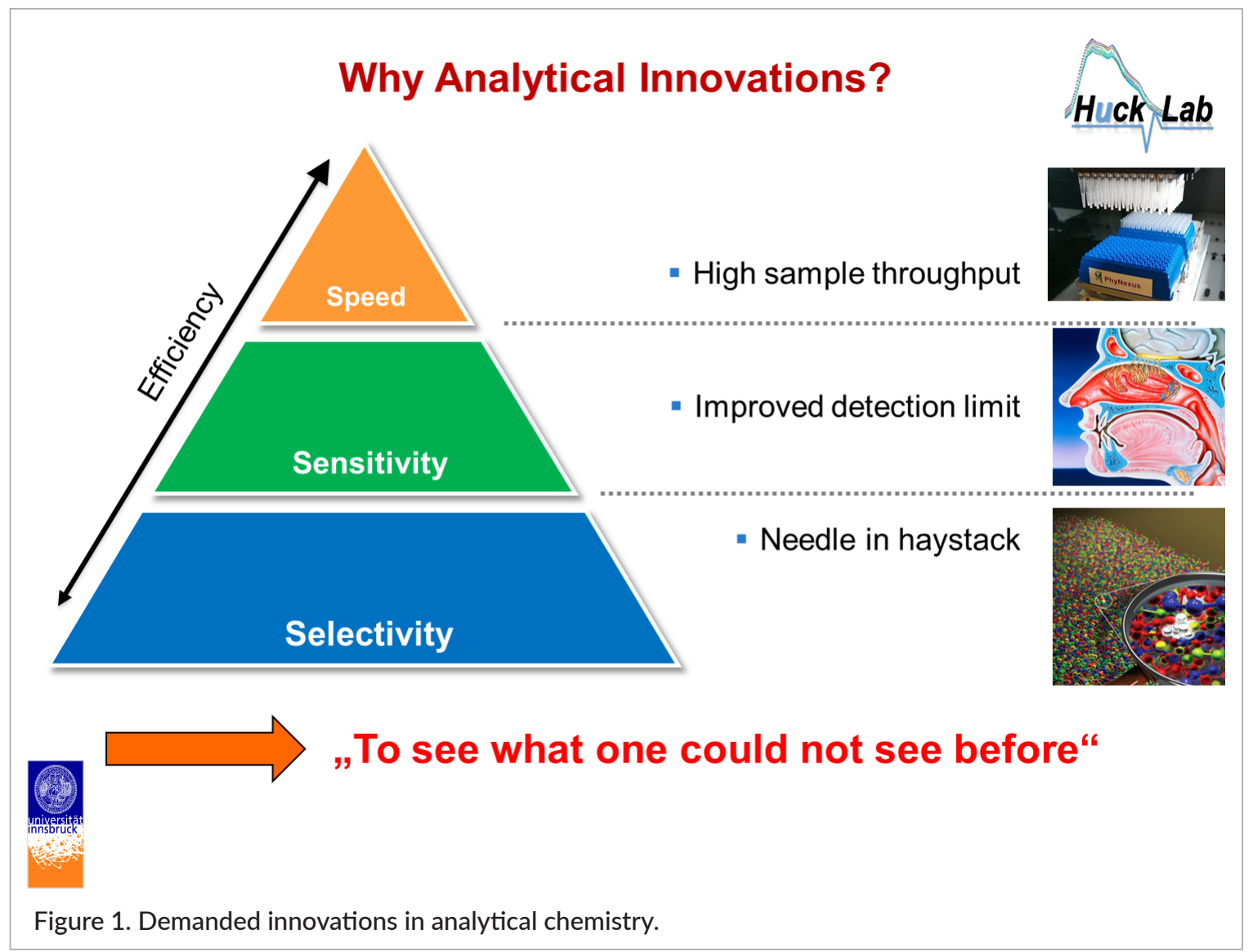

vibrational spectroscopy: The first trend is to achieve higher performance, which requires the implementation of expensive equipment, e.g., laser light sources and sensitive detectors. The second trend is towards miniaturization, which allows cheap and easy analysis at any independent place and/or time by small and portable spectrometric devices, including data transfer via WLAN or Bluetooth (Figure 2). ${ }^{10}$

\section{Miniaturization}

Miniaturization is one of the major trends in NIR spectroscopy. Today, small NIR sensors are either based on micro-electromechanical systems (MEMS) or linear variable tune able filters (LVTF) for appropriate wavelength selection. ${ }^{11}$ Lutz et al. described the modification of an InGaAs diode array detector equipped miniaturized NIR spectrometer enabling the reliable quantification of ethanol blended gasoline. ${ }^{10}$ A transflectance measurement cell was presented, utilizing a thermoelectric cooling (TEC) appliance ensuring thermostatic measurement conditions and a gold-coated spherical mirror as a reflector superior to conventional Spectralon ${ }^{\circledR}$. The testset validated multivariate partial least squares regression (PLSR) model of the measurement mode involving both gold mirror and TEC yielded an $\mathrm{R}_{\text {val }}^{2}$ value of 0.997, a limit of detection (LOD) of $0.68 \% \mathrm{w} / \mathrm{w}$, a limit of quantification ( $\mathrm{LOQ}$ ) of $2.04 \% \mathrm{w} / \mathrm{w}$, a standard error of prediction (SEP) of $0.21 \% \mathrm{w} / \mathrm{w}$ and a ratio performance deviation (RPD) of 15.2. Additionally, the NIR band assignment has been established by employing the vibrational selfconsistent field second order perturbative treatment (PT2-VSCF) and the computationally derived absorption maxima were compared to the experimentally observed data. $^{10}$

As different benchtop and miniaturized spectrometers operate in different wavenumber ranges and resolution, a critical and systematic performance evaluation is needed. For this purpose the workflow presented in Figure 3 can be very helpful, which on the right side performs a detailed comparison based on multivariate data analysis (MVA) also considering two dimensional-correlation spectroscopy (2D-COS) and the determination of the LOD, on the left side quantum chemical calculation can be very helpful checking for 

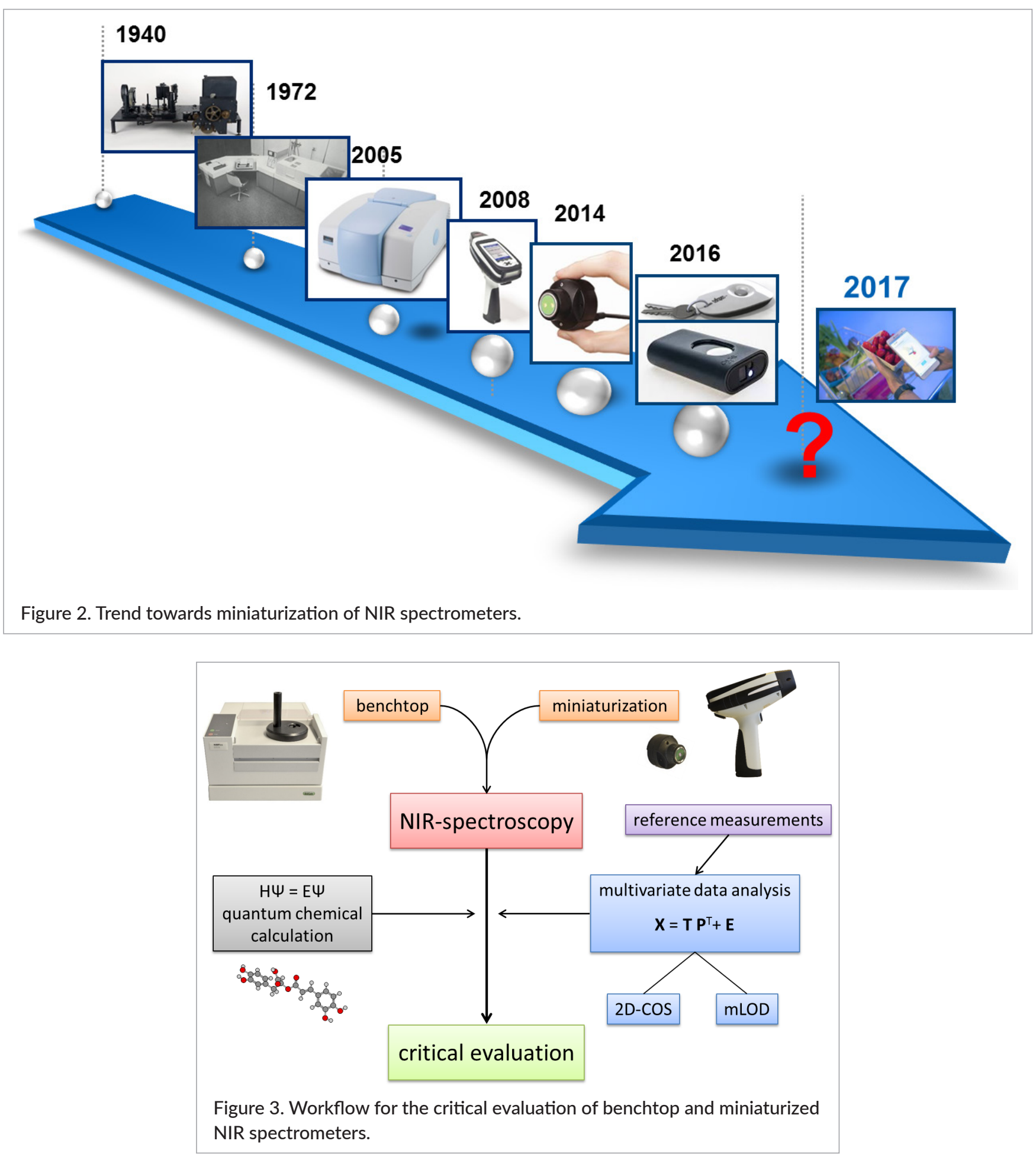

correct band appearance and assignment. Kirchler et al. described the critical evaluation of spectral information of benchtop vs portable NIR spectrometers, conducting quantum chemistry and 2D-COS for a better understanding of PLS regression models of the rosmarinic acid content in Rosmarini folium. ${ }^{12}$ Henn et al. performed a similar study determining the performance of minia- turizations on melamine in milk powder ${ }^{13}$ and compared the sensitivity to artificial spectral errors and multivariate LOD. The best RMSEP values were $0.28 \mathrm{~g} / 100 \mathrm{~g}$, $0.33 \mathrm{~g} / 100 \mathrm{~g}$ and $0.27 \mathrm{~g} / 100 \mathrm{~g}$ for the NIRFlex N-500, the portable/miniaturized spectrometers microPHAZIR and the microNIR2200, respectively. Furthermore, the multivariate LOD interval [LODmin, LODmax] was 
calculated for all the PLSR models unveiling significant differences among the spectrometers showing values of $0.20-0.27 \mathrm{~g} / 100 \mathrm{~g}, 0.28-0.54 \mathrm{~g} / 100 \mathrm{~g}$ and $0.44-$ $1.01 \mathrm{~g} / 100 \mathrm{~g}$ for the NIRFlex $\mathrm{N}-500$, the microPHAZIR and the microNIR2200, respectively. To assess the robustness of all models, artificial introduction of white noise, baseline shift, multiplicative effect, spectral shrink and stretch, stray light and spectral shift were applied. Monitoring the RMSEP as function of the perturbation gave an indication of robustness of the models and helped to compare the performances of the spectrometers. Not taking the additional information from the LOD calculations into account one could falsely assume that all the spectrometers perform equally well which is not the case when the multivariate evaluation and robustness data were considered. These two application examples clearly show the efficiency of the scheme introduced in Figure 3.

\section{Material enhanced infrared spectroscopy (MEIRS)}

Material enhanced infrared spectroscopy (MEIRS) is a method that combines the beneficial properties of solidphase extraction (SPE) and infrared spectroscopy. ${ }^{14,15}$ Petter et al. introduced in 2009 a novel MEIRS approach for the fast and automated quantification of low-density and high-density lipoproteins ( $L D L$ and $H D L$ ) in human serum, ${ }^{14}$ replacing time-consuming routinely applied wet chemical analyses. This MEIRS approach definitely benefits from the automated combined enrichment and measurement approach. Titanium oxide beads were used as an adsorbent for selectively immobilizing LDL and $\mathrm{HDL}$-cholesterol, further analyzing the incubated and washed samples via NIR diffuse reflection spectroscopy. A principal component regression (PCR) model of 24 LDL standards in a range from $500 \mathrm{ppm}$ to $3000 \mathrm{ppm}$ (clinical value is $1500 \mathrm{ppm}$ ) and a partial least squares regression (PLSR) model of $25 \mathrm{HDL}$ standards in a range from $100 \mathrm{ppm}$ to $1000 \mathrm{ppm}$ (clinical value is $400 \mathrm{ppm}$ ) were computed. The regression correlations were $>0.99$ (calibration) and $>0.97$ (validation). The PCR model of the MEIRS based $\mathrm{TiO}_{2}$ - $\mathrm{LDL}$ showed a standard error of estimation (SEE) of $122.80 \mathrm{ppm}$ and a standard error of prediction (SEP) of $121.15 \mathrm{ppm}$ while the PLSR model of $\mathrm{TiO}_{2}-\mathrm{HDL}$ showed $47.70 \mathrm{ppm}$ and $47.14 \mathrm{ppm}$, respec- tively. The MEIRS principle can be easily extrapolated to similar analytical investigations.

\section{Theoretical NIR spectroscopy}

Theoretical NIR spectroscopy based on quantum chemical calculations is becoming more and more important to understand the highly complexity of NIR spectra. ${ }^{16}$ An excellent example for its powerful efficiency is summarized in the following: Grabska et al. described the temperature drift of conformational equilibria of butyl alcohols studied by NIRS and fully anharmonic DFT. ${ }^{17}$ In this contribution four kinds of butyl alcohols, 1-butanol, 2-butanol, iso-butanol and tert-butyl alcohol, were investigated in diluted $(0.1 \mathrm{M}) \mathrm{CCl}_{4}$ solutions. The experimental NIR spectra were accurately reproduced and explained in a fully anharmonic DFT study by means of generalized second-order vibrational perturbation theory (GVPT2). Conformational populations were taken into account in each case. The temperature-dependent NIR spectra of butyl alcohols show changes in the band shape and a blue-shift of the overtone band due to the stretching mode of free $\mathrm{OH}$ group. Intensity decreases with increasing temperature. These effects can be monitored by two dimensional-correlation spectroscopy (2D-COS). In this contribution, the experimental 2D-COS patterns have been successfully reproduced, based on DFT calculated NIR spectra of conformational isomers of the studied molecules and their Boltzmann coefficients over the corresponding temperature range. Thus, the experimentally observed effects are fully reflected in the DFT study, which leads to the conclusion that the main factor in the temperature-dependent spectral changes of $2 \mathrm{vOH}$ band of aliphatic alcohols in the diluted phase, where no self-association occurs, is played by the changes in the relative population of their conformational isomers. In the future, these quantum chemical calculations must be extrapolated to larger molecules.

\section{Application fields}

Application fields discussed include medicinal plants, food, bio- and material analysis (Figure 4). Medicinal plants are becoming more and more important as an alternative to synthetic drugs. In case of food methods, to detect fraud and quality are required. In the bio- and material analysis fields time consuming traditional methods for quality control should get replaced. 


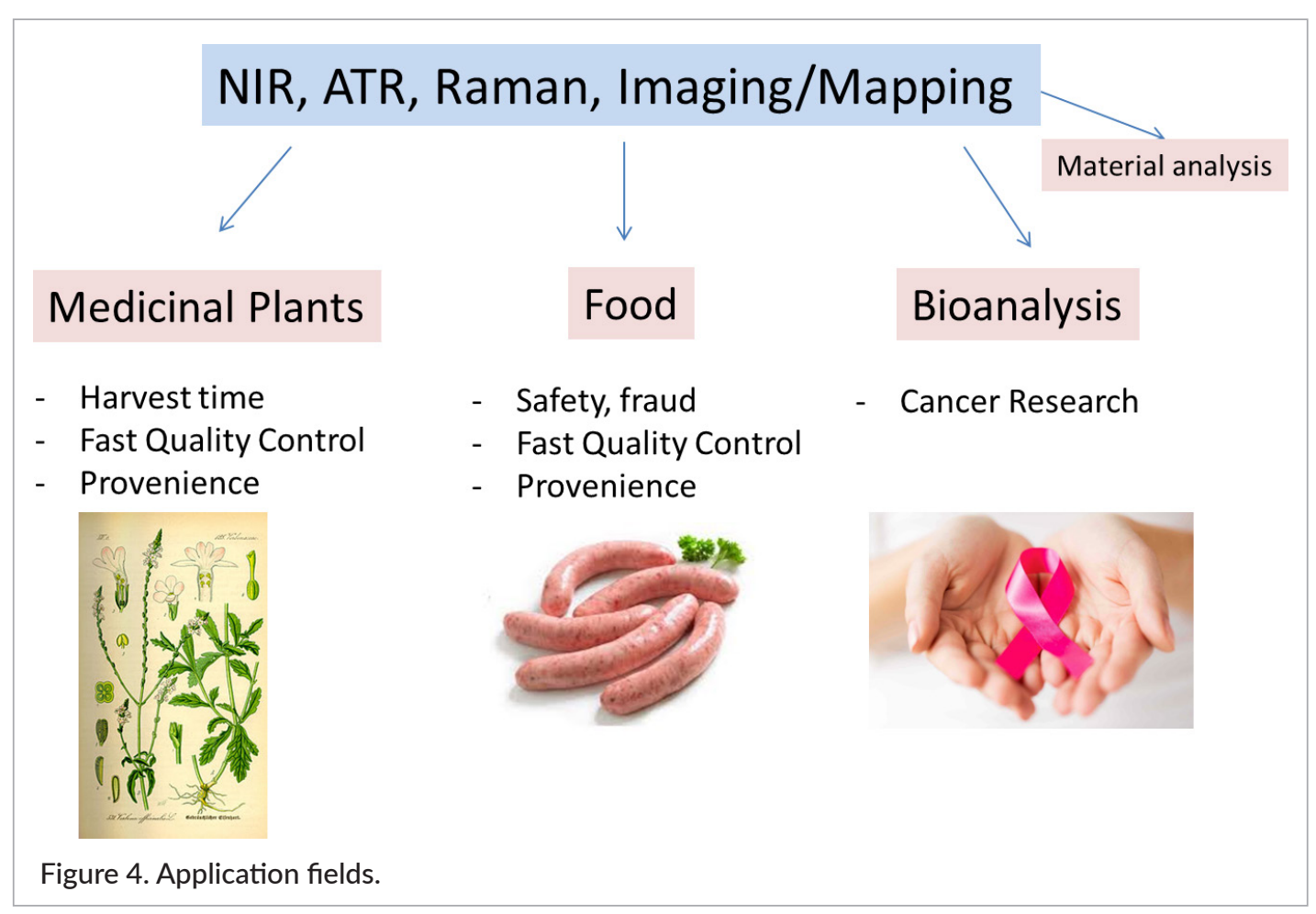

\section{Medicinal plants}

The quality of a medicinal plant is developing during its growth. Therefore, Pezzei et al. investigated the application of benchtop and portable NIR spectrometers for predicting the optimum harvest time of Verbena officinalis. ${ }^{18}$ NIR analyses were performed non-invasively on the fresh plant material based on the quantification of the key constituents verbenalin and verbascoside. Vibrational spectroscopic measurements were performed applying a conventional NIR benchtop device as well as a laboratory independent handheld NIR spectrometer. Quality parameters obtained for the benchtop device revealed that the newly established NIR method enabled reliable quantifications of the main compounds verbenalin and verbascoside related to the dried and fresh plant material. The results of the miniaturized spectrometer revealed that accurate quantitative calibration models could be developed for verbascoside achieving a comparable prediction power to the benchtop device (Table 1). PLS models for verbenalin were less precise suggesting the application of portable devices including a different spectral range and resolution.

The critical performance evaluation of benchtop and miniaturized spectrometers for the quantitative analysis of rosmarinic acid in Rosmarini folium conducting 2D-COS and quantum chemical calculations has already been summarized. ${ }^{12}$ The possibilities employing spectral imaging for medicinal plant quality control have been recently reviewed by Türker-Kaya et al. ${ }^{19}$

\section{Food}

Food scandals and fraud demand new analytical quality control tools. Schmutzler et al. developed a method for detection of pork adulteration in veal product based on NIRS for laboratory, industrial and on-site analysis. ${ }^{20}$ Measurements were also carried out directly through a polymer packaging of the samples and compared to measurements through quartz cuvettes. Meat and fat adulteration could be detected up to the lowest level of contamination (10\%) applying a laboratory setup and the industrial fibre optics setup, regarding measurements through quartz and polymer packaging. Analyses with the on-site setup led to successful separation up to the lowest degree of contamination (10\%, measurement through quartz cuvettes) regarding meat adulteration and up to $20 \%$ and $40 \%$ contamination regarding the fat adulteration performing measurements through quartz cuvettes and through polymer packaging, respectively.

In 2016 Schmutzler et al. developed an automatic sample rotation machine enabling the simultaneous determination of geographical origin and quality characteristics of apples based on NIRS. ${ }^{21,22}$ In this contribu- 


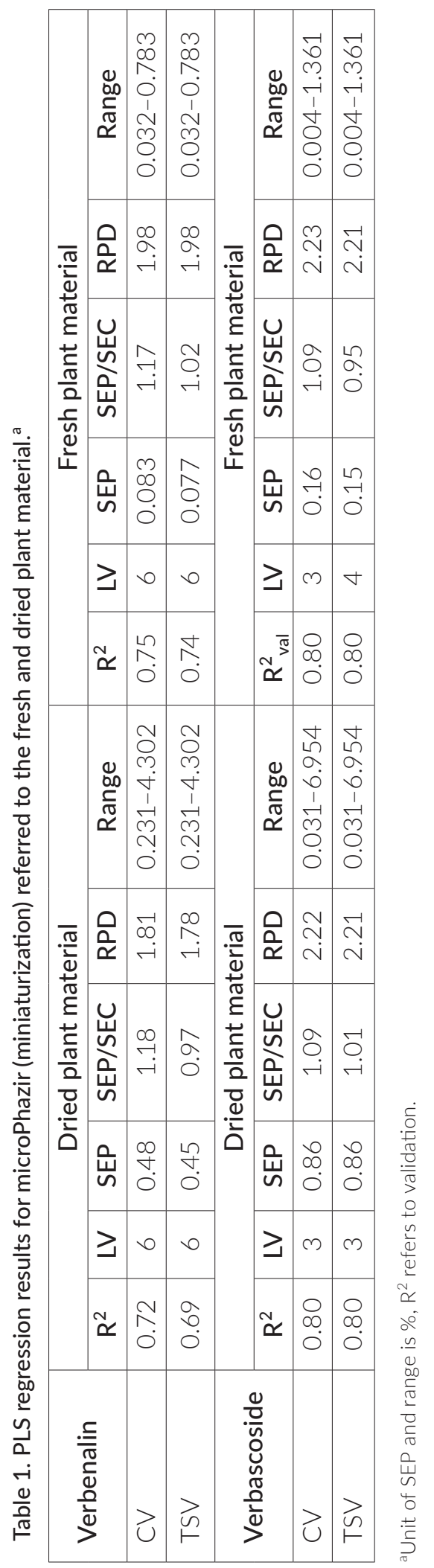

tion the advantages of recording 250 spectra within 40 seconds compared to four point and/or equatorial measurements could be clearly demonstrated. Optimization of spectral parameters via design of experiments (DOE) can be highly helpful. ${ }^{23}$

\section{Bioanalysis}

The application of MEIRS for the fast quantitative analysis of LDL- and HDL-cholesterol has been already summarized. ${ }^{14}$ Ishigaki et al. demonstrated the powerful efficiency of NIRS and NIR imaging for the in vivo monitoring of the growth of fertilized eggs of Medaka Fish (Oryzias latipes). ${ }^{24}$

\section{Material analysis}

NIRS is highly suitable to replace time-consuming traditional analytical methods for the characterization of both chemical and physical (physicochemical) parameters. Märk et al. developed an online process control of a pharmaceutical intermediate in a fluidized-bed drier environment using NIRS in an antibiotics production plant. ${ }^{25}$ For the online process control of the drying process and determination of the ideal drying end point, a continuous NIRS measuring setup was implemented to rapidly and simultaneously gain all essential product information. A bypass system outside the drier combined with a robust process probe proved to provide the best sampling system geometry. Multivariate calibrations for product assay, water content and residual solvent were calculated, optimized and compared with an offline spectrometer. The final root-mean-square error of cross validation (RMSECV) for the process probe could be reduced to $0.81 \%$ for the product assay, $0.25 \%$ for water and $0.06 \%$ for acetone. The laboratory-probe prediction values show good agreement with reference data during the testing period. The established technology is of high importance for the pharmaceutical industry carrying out high-throughput routine analysis because of its advantages in terms of time and cost reductions.

Several NIRS methods have been developed for the characterization of different nano-materials, comprising silica gels, ${ }^{26}$ polymeric monoliths, ${ }^{27}$ fullerenes ${ }^{28}$ and dendrimeres. ${ }^{29}$ Chemical parameters including identification of the material, derivatization control has been accomplished as well as the quantitative analysis of physical parameters including particle size, porosity and surface area. ${ }^{30}$ 


\section{Conclusions}

Since 2010, altogether 290 publications have been registered in the SciFinder database of the ACS dealing with "near infrared spectroscopy in industry", showing a yearly slight increase due to the growing interest towards implementing NIRS in the industrial environment. In the following years, miniaturization and quantum chemical calculations will further push the implementation of NIRS.

\section{References}

1. T. Branigan, The Guardian (2008). https:// www.theguardian.com/world/2008/dec/02/ china?CMP=share_btn_link

2. C.W. Huck, V.A.C. Huck-Pezzei and C.K. Pezzei, Curr. Opin. Food Sci. 10, 32-37 (2016). https://doi. org/10.1016/j.cofs.2016.07.004

3. S. Charlebois, A. Schwab, R. Henn and C.W. Huck, Trends Food Sci. Technol. 50, 211-218 (2016). https:// doi.org/10.1016/j.tifs.2016.02.003

4. N. O'Brien, C.A. Hulse, F. Pfeifer and H.W. Siesler, J. Near Infrared Spectrosc. 21, 299-305 (2013). https:// doi.org/10.1255/jnirs.1063

5. C.W. Huck and G.K. Bonn, J. Chromatogr. A 885, 51-72 (2000). https://doi.org/10.1016/S00219673(00)00333-2

6. A. Greiderer, S.C. Ligon, C.W. Huck and G.K. Bonn, J. Sep. Sci. 32, 2510-2520 (2009). https://doi. org/10.1002/jssc.200900211

7. A. Murauer, R. Bakry, H. Schottenberger, C. Huck and M. Ganzera, Anal. Chim. Acta 963, 136-142 (2017). https://doi.org/10.1016/j.aca.2017.01.048

8. S.M. Valtiner, G.K. Bonn and C.W. Huck, Phytochem. Anal. 19, 359-67 (2008). https://doi.org/10.1002/ pca.1062

9. M. Najam-ul-Haq, M. Rainer, T. Schwarzenauer, C.W. Huck and G.K. Bonn, Anal. Chim. Acta 561, 32-39 (2006). https://doi.org/10.1016/j. aca.2006.01.012

10. O.M.D. Lutz, G.K. Bonn, B.M. Rode and C.W. Huck, Anal. Chim. Acta 826, 61-68 (2014). https://doi. org/10.1016/j.aca.2014.04.002

11. C.W. Huck, GIT Lab. J. 29, 2-5 (2016).

12. C.G. Kirchler, C.K. Pezzei, K.B. Beć, S. Mayr, M. Ishigaki, Y. Ozaki and C.W. Huck, Analyst
142, 455-464 (2017). https://doi.org/10.1039/ C6AN02439D

13. R. Henn, C.G. Kirchler, M.-E. Grossgut and C.W. Huck, Talanta 166, 109-118 (2017). https://doi. org/10.1016/j.talanta.2017.01.035

14. C.H. Petter, N. Heigl, R. Bakry, G.K. Bonn, A. Ritsch and C.W. Huck, Curr. Med. Chem. 16, 4601-4608 (2009). https://doi. org/10.2174/092986709789760625

15. C.H. Petter, Analytik von Biomolekülen Mittels NahInfrarot Spektroskopie-Material Enhanced Infrared Spectroscopy-(MEIRS). Suedwestdeutscher Verlag fuer Hochschulschriften (2009).

16. K.B. Beć, J. Grabska, C.W. Huck, Y. Ozaki and J.P. Hawranek, J. Mol. Liq. 224, 1189-1198 (2016). https://doi.org/10.1016/j.molliq.2016.10.095

17. J. Grabska, K.B. Beć, Y. Ozaki and C.W. Huck, J. Phys. Chem. A 121, 1950-1961 (2017). https://doi. org/10.1021/acs.jpca.7b00646

18. C.K. Pezzei, S.A. Schönbichler, C.G. Kirchler, J. Schmelzer, S. Hussain, V.A. Huck-Pezzei, M. Popp, J. Krolitzek, G.K. Bonn and C.W. Huck, Talanta 169, 70-76 (2017). https://doi.org/10.1016/j. talanta.2017.03.067

19. S. Türker-Kaya and C. Huck, Molecules 22, 168 (2017). https://doi.org/10.3390/molecules22010168

20. M. Schmutzler, A. Beganovic, G. Böhler and C.W. Huck, Food Control 57, 258-267 (2015). https://doi. org/10.1016/j.foodcont.2015.04.019

21. M. Schmutzler and C.W. Huck, Vib. Spectrosc. 72, 97-104 (2014). https://doi.org/10.1016/j.vibspec.2014.02.010

22. M. Schmutzler and C.W. Huck, Food Control 66, 27-37 (2016). https://doi.org/10.1016/j.foodcont.2016.01.026

23. L. De Benedictis and C. Huck, Food Chem. 212, 552-560 (2016). https://doi.org/10.1016/j.foodchem.2016.06.012

24. M. Ishigaki, Y. Yasui, P. Puangchit, S. Kawasaki and Y. Ozaki, Molecules 21, 1003 (2016). https://doi. org/10.3390/molecules21081003

25. J. Märk, M. Karner, M. Andre, J. Rueland and C.W. Huck, Anal. Chem. 82, 4209-4215 (2010).

26. C. Huck, R. Ohmacht, Z. Szabo and G. Bonn, J. Near Infrared Spectrosc. 14, 51 (2006). https://doi. org/10.1255/jnirs.586

27. N. Heigl, A. Greiderer, C.H. Petter, O. Kolomiets, H.W. Siesler, M. Ulbricht, G.K. Bonn and C.W. Huck, 
Anal. Chem. 80, 8493-8500 (2008). https://doi.

org/10.1021/ac8013059

28. R.M. Vallant, Z. Szabo, S. Bachmann, R. Bakry, M. Najam-ul-Haq, M. Rainer, N. Heigl, C. Petter, C.W. Huck and G.K. Bonn, Anal. Chem. 79, 81448153 (2007). https://doi.org/10.1021/ac0712392

29. N. Heigl, S. Bachmann, C.H. Petter, M. MarchettiDeschmann, G. Allmaier, G.K. Bonn and C.W. Huck, Anal. Chem. 81, 5655-5662 (2009). https://doi. org/10.1021/ac900375z

30. C.W. Huck, C. Pezzei, V.A. Huck-Pezzei, J.D. Pallua, S.A. Schoenbichler, L.K. Bittner and G.K. Bonn, Recent Pat. Nanotechnol. 6, 135-141 (2012). https:// doi.org/10.2174/187221012800270171 